\title{
Bi Ideals of Nearness Semirings
}

\author{
Özlem Tekin ${ }^{1 *}$ \\ $1^{*}$ Adiyaman University, Faculty of Arts and Sciences, Department of Mathematics, Adiyaman, Turkey, (ORCID: 0000-0002-1721-1053) \\ umduozlem42@gmail.com
}

(1st International Conference on Applied Engineering and Natural Sciences ICAENS 2021, November 1-3, 2021)

(DOI: 10.31590/ejosat.973355)

ATIF/REFERENCE: Tekin, Ö. (2021). Bi Ideals of Nearness Semirings. European Journal of Science and Technology, (28), 11-15.

\begin{abstract}
$\mathrm{Bi}$ ideals are the generalisation of quasi ideals. In this article, it is defined that the notion of bi-ideals in semirings on weak nearness approximation spaces. Afterwards, it is explained that some of the concepts and definitions related to the subject. Also, it is given that the definition of nearness $m$-bi ideals and nearness $(m, n)$-quasi ideals. Thus, we examine the relationship between nearness $m$-bi ideals and nearness $(\mathrm{m}, \mathrm{n})$-quasi ideals .
\end{abstract}

Keywords: Nearness approximation space, Semirings, Nearness semiring, Quasi ideals, Bi ideals.

\section{Yakınlık Yarı Halkalarının Bi İdealleri}

Öz

Bi idealler, quasi ideallerin bir genelleştirmesidir. Bu çalışmada, zayıf yakınlık yaklaşım uzaylarında bi-idealler kavramı tanımlandı. Daha sonra, konuyla ilgili bazı tanımlar ve kavramlar açıklandı. Ayrıca, yakınlık $m$-bi idealleri ve yakınlık $(m, n)-$ quasi ideallerinin tanımları verildi. Böylece, yakınlık m-bi idealleri ve yakınlık (m,n)-quasi ideallerinin aralarındaki ilişkiyi inceledik.

Anahtar Kelimeler: Yakınlık Yaklaşım Uzayları, Yarı Halkalar, Yakınlık Yarı Halkaları, Quasi idealler, Bi idealler.

\footnotetext{
* Corresponding Author: umduozlem42@,gmail.com
} 


\section{Introduction}

Peters studied near sets theory that is a generalisation of rough sets [1] in 2002. Peters gaved an indiscernibility relation by utilizing the features of the objects to determine the nearness of the objects [2]. Afterwards, he generalised approach theory in the study of the nearness of non-empty sets which are similar to each other [3], [4]. İnan and Öztürk introduced the notion of nearness groups [5]. Therefore, other approaches have been studied in [6], [7], [8].

The concept of semiring theory was defined by Vandier [9] in 1934 and many mathematicians proved important properties for semiring theory. Especially, semirings are very important for determinants and matrices. One of the most important notion for semirings is ideals. Henriksen and Shabir et al. [10] studied ideals for semirings. In 1952, the notion of bi ideals for semigroups was defined by Good and Hughes [11]. Afterwards, Lajos and Szasz introduced theory of bi ideals in rings and semirings [12]. Biideals are a special situation of $(m, n)$ ideal. Tekin defined quasi ideals in semirings on weak nearness approximation spaces [13].

In this article, bi ideals in semirings are defined and some of the concepts and definitions on weak nearness approximation spaces are explained. Furthermore, we study some basic properties of bi ideals.

\section{Preliminaries}

An object characterization is given by means of a tuple of function values $\Phi(x)$ deal with an object $x \in X . B \subseteq F$ is a set of probe functions and these functions stand for features of sample objects $X \subseteq O$. Let $\varphi_{i} \in B$, that is $\varphi_{i}: \mathcal{O} \rightarrow \mathbb{R}$. The functions showing object features supply a basis for, $\Phi: \mathcal{O} \rightarrow \mathbb{R}^{L}, \Phi(x)=$ $\left(\varphi_{1}(x), \varphi_{2}(x), \ldots, \varphi_{L}(x)\right)$ a vector consisting of measurements deal with each functional value $\varphi_{i}(x)$, where the description length $|\Phi|=L([2])$.

The choice of functions $\varphi_{i} \in B$ is very important by using to determine sample objects. Each $\varphi$ shows a descriptive pattern of an object. The difference $\varphi$ means to a description of the indiscernibility relation " $\sim_{B}$ " defined by Peters in [2]. $B_{r}$ is probe functions in $B$ for $r \leq|B|$.

\section{Definition 2.1 [2]}

$$
\sim_{B}=\left\{\left(x, x^{\prime}\right) \in \mathcal{O} \times \mathcal{O} \mid \triangle_{\varphi_{i}}=0, \forall \varphi_{i} \in B, B \subseteq \mathcal{F}\right\}
$$

means indiscernibility relation on $\mathcal{O}$, where description length $i \leq$ $|\Phi| . \sim_{B_{r}}$ is also indiscernibility relation determined by utilizing $B_{r}$.

Near equivalence class is stated as $[x]_{B_{r}}=\left\{x^{\prime} \in\right.$ $\left.\mathcal{O} \mid x \sim_{B_{r}} x^{\prime}\right\}$. After attaining near equivalence classes, quotient set $\mathcal{O} / \sim_{B_{r}}=\left\{[x]_{B_{r}} \mid x \in \mathcal{O}\right\}=\xi_{\mathcal{O}, B_{r}}$ and set of partitions $N_{r}(B)=$ $\left\{\xi_{\mathcal{O}, B_{r}} \mid B_{r} \subseteq B\right\}$ can be found. By using near equivalence classes, $N_{r}(B)^{*} X=\bigcup_{[x]_{B_{r}} \cap X \neq \varnothing}[x]_{B_{r}}$ upper approximation set can be got.

\section{Definition 2.2 [14]}

Let $\mathcal{O}$ be a set of sample objects, $\mathcal{F}$ a set of the probe functions, $\sim_{B_{r}}$ an indiscernibility relation, and $N_{r}(B)$ a collection of partitions. Then, $\left(\mathcal{O}, \mathcal{F}, \sim_{B_{r}}, N_{r}(B)\right)$ is called a weak nearness approximation space.
Theorem 2.1 [14]

Let $\left(\mathcal{O}, \mathcal{F}, \sim_{B_{r}}, N_{r}(B)\right)$ be a weak nearness approximation space and $X, Y \subset \mathcal{O}$. Then the followings hold:

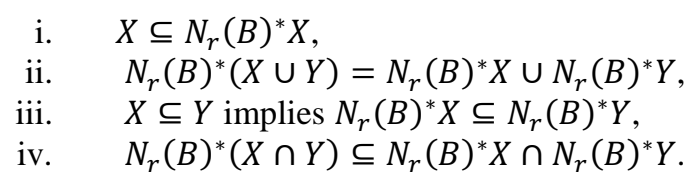

Afterward, $\mathcal{O}$ means a $\left(\mathcal{O}, \mathcal{F}, \sim_{B_{r}}, N_{r}(B)\right)$ is weak near approximation spaces unless otherwise said.

Definition 2.3 [6]

Let $S$ be a nearness semigroup. For all $x \in S$,

there exists an element $e \in N_{r}(B)^{*} S$ such that

$x \cdot e=e \cdot x=x$ hold, then $(S, \cdot)$ is called a nearness monoid.

Definition 2.4 [6]

Let $S \subseteq \mathcal{O}$. Then, $S$ is called a semiring on weak near approximation spaces $\mathcal{O}$ if the following properties hold: element 0 ,

$\left.N S R_{1}\right)(S,+)$ is an abelian monoid on $\mathcal{O}$ with identity

$\left.N S R_{2}\right)(S, \cdot)$ is a monoid on $\mathcal{O}$ with identity element $1_{S}$,

$N S R_{3}$ ) for all $x, y, z \in S$ such that

$x \cdot(y+z)=(x \cdot y)+(x \cdot z)$ and $(x+y) \cdot z=(x \cdot z)+(y$

$z$ ) hold in $N_{r}(B)^{*} S$,

$N S R_{4}$ ) for all $x \in S$ such that

$$
0 \cdot x=0=x \cdot 0
$$

hold in $N_{r}(B)^{*} S$,

$$
\left.N S R_{5}\right) 1 \neq 0 \text {. }
$$

Theorem 2.2 [6]

Let $\left(\mathcal{O}, \mathcal{F}, \sim_{B_{r}}, N_{r}(B)\right)$ be a weak nearness approximation space and $X, Y \subset \mathcal{O}$. Then the followings hold:

$$
\text { i. } \quad\left(N_{r}(B)^{*} X\right)+\left(N_{r}(B)^{*} Y\right) \subseteq N_{r}(B)^{*}(X+Y)
$$$$
\text { ii. } \quad\left(N_{r}(B)^{*} X\right) \cdot\left(N_{r}(B)^{*} Y\right) \subseteq N_{r}(B)^{*}(X \cdot Y) \text {. }
$$

Definition 2.5 [6]

Let $S$ be a nearness semiring, and $A$ is a non-empty subset of $S$.

i. $\quad A$ is called a subsemiring of $S$, if $A+A \subseteq N_{r}(B)^{*} A$ and $A \cdot A \subseteq N_{r}(B)^{*} A$.

ii. $\quad A$ is called an upper-near subsemiring of $S$, if $\left(N_{r}(B)^{*} A\right)+$ $\left(N_{r}(B)^{*} A\right) \subseteq N_{r}(B)^{*} A$ and $\left(N_{r}(B)^{*} A\right) \cdot\left(N_{r}(B)^{*} A\right) \subseteq$ $N_{r}(B)^{*} A$.

Definition 2.6 [6]

Let $S$ be a nearness semiring, and $A$ be a subsemigroup of $S$, where $A \neq S$.

i. $\quad A$ is called a right (left) ideals of $S$, if $A \cdot S \subseteq N_{r}(B)^{*} A(S$. $\left.A \subseteq N_{r}(B)^{*} A\right)$.

ii. $\quad A$ is called an upper-near right (left) ideals of $S$, if $\left(N_{r}(B)^{*} A\right) \cdot S \subseteq N_{r}(B)^{*} A\left(S \cdot\left(N_{r}(B)^{*} A\right) \subseteq N_{r}(B)^{*} A\right)$.

Definition 2.7 [15]

Let $M$ be a semiring and $A$ be a non-empty subset of semiring $M$. 
If $A$ is a subsemigroup of $M$ and $B M B \subseteq B$, then $B$ is called a bi ideal of $M$.

\section{Definition 2.8 [13]}

Let $S$ be a nearness semiring and $Q$ be non-empty subset of $S$, where $Q \neq S$. $Q$ is called quasi-ideal of $S$ if $Q$ is a subnearness semigroup of $S$ and $Q S \cap S Q \subseteq N_{r}(B)^{*} Q$.

\section{Lemma 2.1 [13]}

Let $S$ be a nearness semiring. If $S$ is commutative, then each quasi-ideal of $S$ is two-sided ideal of $S$.

\section{Bi Ideals of Nearness Semirings}

\section{Definition 3.9}

Let $S$ be a nearness semiring and $A$ is a subsemigroup of $S$, where $A \subseteq S$.

i. $\quad A$ is called bi ideal of $S$ if $A M A \subseteq N_{r}(B)^{*} A$.

ii. $A$ is called an upper-near bi ideal of $S$ if $\left(N_{r}(B)^{*} A\right) S\left(N_{r}(B)^{*} A\right) \subseteq N_{r}(B)^{*} A$.

\section{Example 3.1}

Let $\mathcal{O}=\{a, b, c, d, e, f, g, h, i, j\}$ be a set of perceptual objects where $r=1, B=\left\{\varphi_{1}, \varphi_{2}, \varphi_{3}\right\} \subseteq \mathcal{F}$ be a set of probe functions. Let $S=\{c, d, e, f\} \subset \mathcal{O}$. Probe functions'values

$$
\begin{aligned}
& \varphi_{1}: \mathcal{O} \rightarrow V_{1}=\left\{\alpha_{1}, \alpha_{2}, \alpha_{3}, \alpha_{4}, \alpha_{5}\right\} \\
& \varphi_{2}: \mathcal{O} \rightarrow V_{2}=\left\{\alpha_{2}, \alpha_{3}, \alpha_{4}, \alpha_{5}, \alpha_{6}\right\} \\
& \varphi_{3}: \mathcal{O} \rightarrow V_{3}=\left\{\alpha_{2}, \alpha_{4}, \alpha_{6}, \alpha_{7}, \alpha_{8}\right\}
\end{aligned}
$$

are presented in the following table:

\begin{tabular}{c|cccccccccc} 
& $a$ & $b$ & $c$ & $d$ & $e$ & $f$ & $g$ & $h$ & $i$ & $j$ \\
\hline$\varphi_{1}$ & $\alpha_{1}$ & $\alpha_{2}$ & $\alpha_{3}$ & $\alpha_{2}$ & $\alpha_{3}$ & $\alpha_{4}$ & $\alpha_{2}$ & $\alpha_{1}$ & $\alpha_{1}$ & $\alpha_{5}$ \\
$\varphi_{2}$ & $\alpha_{2}$ & $\alpha_{3}$ & $\alpha_{2}$ & $\alpha_{4}$ & $\alpha_{3}$ & $\alpha_{4}$ & $\alpha_{5}$ & $\alpha_{2}$ & $\alpha_{6}$ & $\alpha_{5}$ \\
$\varphi_{3}$ & $\alpha_{2}$ & $\alpha_{4}$ & $\alpha_{6}$ & $\alpha_{6}$ & $\alpha_{7}$ & $\alpha_{7}$ & $\alpha_{2}$ & $\alpha_{4}$ & $\alpha_{8}$ & $\alpha_{8}$
\end{tabular}

Now, we find the near equivalence classes according to the indiscernibility relation $\sim_{B_{r}}$ of elements in $\mathcal{O}$ :

$$
\begin{gathered}
{[a]_{\varphi_{1}}=\left\{x \in \mathcal{O} \mid \varphi_{1}(x)=\varphi_{1}(a)=\alpha_{1}\right\}=\{a, h, i\}=[h]_{\varphi_{1}}} \\
=[i]_{\varphi_{1}} \\
{[b]_{\varphi_{1}}=\left\{x \in \mathcal{O} \mid \varphi_{1}(x)=\varphi_{1}(b)=\alpha_{2}\right\}=\{b, d, g\}} \\
=[d]_{\varphi_{1}}=[g]_{\varphi_{1}}, \\
{[c]_{\varphi_{1}}=\left\{x \in \mathcal{O} \mid \varphi_{1}(x)=\varphi_{1}(c)=\alpha_{3}\right\}=\{c, e\}} \\
=[e]_{\varphi_{1}}, \\
{[f]_{\varphi_{1}}=\left\{x \in \mathcal{O} \mid \varphi_{1}(x)=\varphi_{1}(f)=\alpha_{4}\right\}=\{f\},} \\
{[j]_{\varphi_{1}}=\left\{x \in \mathcal{O} \mid \varphi_{1}(x)=\varphi_{1}(j)=\alpha_{5}\right\}=\{j\} .}
\end{gathered}
$$

Then, we have that $\xi_{\varphi_{1}}=\left\{[a]_{\varphi_{1}},[b]_{\varphi_{1}},[c]_{\varphi_{1}},[f]_{\varphi_{1}},[j]_{\varphi_{1}}\right\}$.

$$
\begin{gathered}
{[a]_{\varphi_{2}}=\left\{x \in \mathcal{O} \mid \varphi_{2}(x)=\varphi_{2}(a)=\alpha_{1}\right\}=\{a, c, h\}} \\
\quad=[c]_{\varphi_{2}}=[h]_{\varphi_{2}}, \\
{[b]_{\varphi_{2}}=\left\{x \in \mathcal{O} \mid \varphi_{2}(x)=\varphi_{2}(b)=\alpha_{3}\right\}=\{b, e\}} \\
\quad=[e]_{\varphi_{2}}, \\
{[d]_{\varphi_{2}}=\left\{x \in \mathcal{O} \mid \varphi_{2}(x)=\varphi_{2}(d)=\alpha_{4}\right\}=\{d, f\}} \\
\quad=[f]_{\varphi_{2}}, \\
{[g]_{\varphi_{2}}=\left\{x \in \mathcal{O} \mid \varphi_{2}(x)=\varphi_{2}(g)=\alpha_{5}\right\}=\{g, j\}} \\
\quad=[j]_{\varphi_{2}}, \\
{[i]_{\varphi_{2}}=\left\{x \in \mathcal{O} \mid \varphi_{2}(x)=\varphi_{2}(i)=\alpha_{6}\right\}=\{i\} .}
\end{gathered}
$$

We attain that $\xi_{\varphi_{2}}=\left\{[a]_{\varphi_{2}},[b]_{\varphi_{2}},[d]_{\varphi_{2}},[g]_{\varphi_{2}},[i]_{\varphi_{2}}\right\}$

$$
\begin{gathered}
{[a]_{\varphi_{3}}=\left\{x \in \mathcal{O} \mid \varphi_{3}(x)=\varphi_{3}(a)=\alpha_{2}\right\}=\{a, g\}} \\
=[g]_{\varphi_{3}}, \\
{[b]_{\varphi_{3}}=\left\{x \in \mathcal{O} \mid \varphi_{3}(x)=\varphi_{3}(b)=\alpha_{4}\right\}=\{b, h\}} \\
=[h]_{\varphi_{3}}, \\
{[c]_{\varphi_{3}}=\left\{x \in \mathcal{O} \mid \varphi_{3}(x)=\varphi_{3}(c)=\alpha_{6}\right\}=\{c, d\}} \\
\quad=[d]_{\varphi_{3}}, \\
{[e]_{\varphi_{3}}=\left\{x \in \mathcal{O} \mid \varphi_{3}(x)=\varphi_{3}(e)=\alpha_{7}\right\}=\{e, f\}} \\
\quad=[f]_{\varphi_{3}}, \\
{[i]_{\varphi_{3}}=\left\{x \in \mathcal{O} \mid \varphi_{3}(x)=\varphi_{3}(i)=\alpha_{8}\right\}=\{i, j\}} \\
\quad=[j]_{\varphi_{3}} .
\end{gathered}
$$

From here, we get that $\xi_{\varphi_{3}}=\left\{[a]_{\varphi_{3}},[b]_{\varphi_{3}},[c]_{\varphi_{3}},[e]_{\varphi_{3}},[i]_{\varphi_{3}}\right\}$ Consequently, a set of partitions of $\mathcal{O}$ is $N_{r}(B)=\left\{\xi_{\varphi_{1}}, \xi_{\varphi_{2}}, \xi_{\varphi_{3}}\right\}$ for $r=1$. Hence,

$[d]_{\varphi_{2}} \cup[c]_{\varphi_{3}} \cup[e]_{\varphi_{3}}$

$$
\begin{aligned}
& N_{1}(B)^{*} S=\bigcup_{[x]_{\varphi_{i}} \cap S \neq \varnothing}[x]_{\varphi_{i}} \\
& =[b]_{\varphi_{1}} \cup[c]_{\varphi_{1}} \cup[f]_{\varphi_{1}} \cup[a]_{\varphi_{2}} \cup[b]_{\varphi_{2}} \cup
\end{aligned}
$$

$$
=\{a, b, c, d, e, f, g, h\} .
$$

Taking operation tables for $S$ in the following tables:

\begin{tabular}{l|llll}
+ & $c$ & $d$ & $e$ & $f$ \\
\hline$c$ & $d$ & $e$ & $f$ & $g$ \\
$d$ & $e$ & $f$ & $g$ & $b$ \\
$e$ & $f$ & $g$ & $b$ & $c$ \\
$f$ & $g$ & $b$ & $c$ & $d$ \\
$\cdot$ & $c$ & $d$ & $e$ & $f$ \\
\hline$c$ & $c$ & $d$ & $e$ & $f$ \\
$d$ & $d$ & $f$ & $b$ & $d$ \\
$e$ & $e$ & $b$ & $e$ & $b$ \\
$f$ & $f$ & $d$ & $b$ & $f$
\end{tabular}


In this case, $(S,+, \cdot)$ is a nearness semiring. Let take $A=\{d, e, f\}$ is subset of $S$.

$$
\begin{gathered}
N_{1}(B)^{*} A=\cup_{[x]_{\varphi_{i}} \cap Q \neq \varnothing}[x]_{\varphi_{i}} \\
=[b]_{\varphi_{1}} \cup[c]_{\varphi_{1}} \cup[f]_{\varphi_{1}} \cup[b]_{\varphi_{2}} \cup[d]_{\varphi_{2}} \cup \\
{[c]_{\varphi_{3}} \cup[e]_{\varphi_{3}}=\{b, c, d, e, f, g\} .}
\end{gathered}
$$

Since, $A$ is a subsemigroup of $S$ and $A S A \subseteq N_{r}(B)^{*} A, A$ is a bi ideal of nearness semiring $S$.

\section{Lemma 3.2}

Let $S$ be a nearness semiring and $A$ be a non-empty subset of $S$. If $S$ is commutative and $N_{r}(B)^{*}\left(N_{r}(B)^{*} A\right)=N_{r}(B)^{*} A$, then each quasi ideal of $S$ is bi ideal of $S$.

Proof. Let $S$ be a commutative nearness semiring and $A$ be a quasi ideal of $S . \quad A S A=(A S A) \cap(A S A)=A(S A) \cap(A S) A \subseteq$ $S(S A) \cap(A S) S \subseteq(S S) A \cap A(S S)$ since $S$ is a nearness semiring. Afterward, $(S S) A \cap A(S S) \subseteq N_{r}(B)^{*} S N_{r}(B)^{*} A \cap$ $N_{r}(B)^{*} A N_{r}(B)^{*} S \subseteq N_{r}(B)^{*}(S A) \cap N_{r}(B)^{*}(A S)$ by Theorem 1 and Theorem 2.(ii). In this case, $N_{r}(B)^{*}(S A) \cap N_{r}(B)^{*}(A S) \subseteq$ $N_{r}(B)^{*}\left(N_{r}(B)^{*} A\right) \cap N_{r}(B)^{*}\left(N_{r}(B)^{*} A\right)=N_{r}(B)^{*}\left(N_{r}(B)^{*} A\right)=$ $N_{r}(B)^{*} A$ from Lemma 1. Hence, $A S A \subseteq N_{r}(B)^{*} A$ and $A$ is a nearness bi ideal of $S$.

\section{Theorem 3. 3}

Let $S$ be a nearness semiring and $A$ be a non-empty subset of $S$. Each right or left nearness ideal of $S$ is a nearness bi ideal of $S$ if $N_{r}(B)^{*} A$ is grupoid.

Proof. Let $A$ be left nearness ideal of $S$. In this case, $S A \subseteq$ $N_{r}(B)^{*} A$. Then, $A S A \subseteq A(S A) \subseteq\left(N_{r}(B)^{*} A\right)\left(N_{r}(B)^{*} A\right)$ by Theorem 1. Then, $\left(N_{r}(B)^{*} A\right)\left(N_{r}(B)^{*} A\right) \subseteq N_{r}(B)^{*} A$ since $N_{r}(B)^{*} A$ is grupoid. Hence, $A S A \subseteq N_{r}(B)^{*} A$ and $A$ is a nearness bi ideal of $S$.

Similarly, $A$ be right nearness ideal. In this way, $A S \subseteq N_{r}(B)^{*} A$. Then, $A S A \subseteq(A S) A \subseteq\left(N_{r}(B)^{*} A\right)\left(N_{r}(B)^{*} A\right)$ by Theorem 1 . Thus, $\quad\left(N_{r}(B)^{*} A\right)\left(N_{r}(B)^{*} A\right) \subseteq N_{r}(B)^{*} A$ since $N_{r}(B)^{*} A$ is grupoid. Afterward, $A S A \subseteq N_{r}(B)^{*} A$ and $A$ is a nearness bi ideal of $S$.

\section{Lemma 3.3}

Let $S$ be a nearness semiring and $A$ be a non-empty subset of $S$. Every bi ideal of $S$ is an upper-near bi ideal of $S$ if $N_{r}(B)^{*}\left(N_{r}(B)^{*} A\right)=N_{r}(B)^{*} A$.

Proof. Let $S$ be a nearness semiring and $A$ is a bi ideal of $S$. $\left(N_{r}(B)^{*} A\right) S\left(N_{r}(B)^{*} A\right) \subseteq\left(N_{r}(B)^{*} A\right)\left(N_{r}(B)^{*} S\right)\left(N_{r}(B)^{*} A\right)$ by Theorem 1. Then, $\left(N_{r}(B)^{*} A\right)\left(N_{r}(B)^{*} S\right)\left(N_{r}(B)^{*} A\right) \subseteq$ $N_{r}(B)^{*}(A S) N_{r}(B)^{*} A$ from Theorem 2.(ii). Again, by Theorem 2.(ii), $N_{r}(B)^{*}(A S) N_{r}(B)^{*} A \subseteq N_{r}(B)^{*}(A S A)$. Since $A$ is a bi ideal of $S$, we get that $N_{r}(B)^{*}(A S A) \subseteq N_{r}(B)^{*}\left(N_{r}(B)^{*} A\right)=N_{r}(B)^{*} A$. Hence, $\left(N_{r}(B)^{*} A\right) S\left(N_{r}(B)^{*} A\right) \subseteq N_{r}(B)^{*} A$ and $A$ is an uppernear bi ideal of $S$.

\section{Corollary 3.1}

Let $S$ be a nearness semiring. If $S$ is commutative and $N_{r}(B)^{*}\left(N_{r}(B)^{*} A\right)=N_{r}(B)^{*} A$, then each quasi ideal is an uppernear bi ideal.

\section{Example 3.2}

Let $\mathcal{O}=\{a, b, c, d, e, f, g, h, i, j\}$ be a set of perceptual objects where $r=1, B=\left\{\varphi_{1}, \varphi_{2}, \varphi_{3}\right\} \subseteq \mathcal{F}$ be a set of probe functions.
Let $S=\{c, d, e, f\} \subset \mathcal{O}$. Probe functions'values

$$
\begin{aligned}
& \varphi_{1}: \mathcal{O} \rightarrow V_{1}=\left\{\alpha_{1}, \alpha_{2}, \alpha_{3}, \alpha_{4}, \alpha_{5}\right\}, \\
& \varphi_{2}: \mathcal{O} \rightarrow V_{2}=\left\{\alpha_{1}, \alpha_{3}, \alpha_{4}, \alpha_{5}, \alpha_{6}\right\}, \\
& \varphi_{3}: \mathcal{O} \rightarrow V_{3}=\left\{\alpha_{2}, \alpha_{3}, \alpha_{4}, \alpha_{5}, \alpha_{6}\right\}
\end{aligned}
$$

are presented in the table below:

\begin{tabular}{c|cccccccccc} 
& $a$ & $b$ & $c$ & $d$ & $e$ & $f$ & $g$ & $h$ & $i$ & $j$ \\
\hline$\varphi_{1}$ & $\alpha_{1}$ & $\alpha_{2}$ & $\alpha_{3}$ & $\alpha_{3}$ & $\alpha_{2}$ & $\alpha_{4}$ & $\alpha_{4}$ & $\alpha_{1}$ & $\alpha_{4}$ & $\alpha_{5}$ \\
$\varphi_{2}$ & $\alpha_{4}$ & $\alpha_{1}$ & $\alpha_{4}$ & $\alpha_{4}$ & $\alpha_{3}$ & $\alpha_{3}$ & $\alpha_{1}$ & $\alpha_{5}$ & $\alpha_{6}$ & $\alpha_{5}$ \\
$\varphi_{3}$ & $\alpha_{2}$ & $\alpha_{3}$ & $\alpha_{2}$ & $\alpha_{3}$ & $\alpha_{4}$ & $\alpha_{5}$ & $\alpha_{4}$ & $\alpha_{6}$ & $\alpha_{6}$ & $\alpha_{6}$
\end{tabular}

Now, we find the near equivalence classes according to the indiscernibility relation $\sim_{B_{r}}$ of elements in $\mathcal{O}$ :

$[a]_{\varphi_{1}}=\left\{x \in \mathcal{O} \mid \varphi_{1}(x)=\varphi_{1}(a)=\alpha_{1}\right\}=\{a, h\}$

$=[h]_{\varphi_{1}}$,

$$
\begin{gathered}
{[b]_{\varphi_{1}}=\left\{x \in \mathcal{O} \mid \varphi_{1}(x)=\varphi_{1}(b)=\alpha_{2}\right\}=\{b, e\}} \\
=[e]_{\varphi_{1}} \\
{[c]_{\varphi_{1}}=\left\{x \in \mathcal{O} \mid \varphi_{1}(x)=\varphi_{1}(c)=\alpha_{3}\right\}=\{c, d\}} \\
{[f]_{\varphi_{1}}=\left\{x \in \mathcal{O} \mid \varphi_{1}(x)=\varphi_{1}(f)=\alpha_{4}\right\}=\{f, g, i\}=[g]_{\varphi_{1}},} \\
=[i]_{\varphi_{1}}, \\
{[j]_{\varphi_{1}}=\left\{x \in \mathcal{O} \mid \varphi_{1}(x)^{\prime}=\varphi_{1}(j)=\alpha_{5}\right\}=\{j\} .}
\end{gathered}
$$

Then, we have that $\xi_{\varphi_{1}}=\left\{[a]_{\varphi_{1}},[b]_{\varphi_{1}},[c]_{\varphi_{1}},[f]_{\varphi_{1}},[j]_{\varphi_{1}}\right\}$.

$$
\begin{gathered}
{[a]_{\varphi_{2}}=\left\{x \in \mathcal{O} \mid \varphi_{2}(x)=\varphi_{2}(a)=\alpha_{4}\right\}=\{a, c, d\}} \\
=[c]_{\varphi_{2}}=[d]_{\varphi_{2}} \\
{[b]_{\varphi_{2}}=\left\{x \in \mathcal{O} \mid \varphi_{2}(x)=\varphi_{2}(b)=\alpha_{1}\right\}=\{b, g\}} \\
=[g]_{\varphi_{2}} \\
{[e]_{\varphi_{2}}=\left\{x \in \mathcal{O} \mid \varphi_{2}(x)=\varphi_{2}(e)=\alpha_{3}\right\}=\{e, f\}} \\
=[f]_{\varphi_{2}}, \\
{[h]_{\varphi_{2}}=\left\{x \in \mathcal{O} \mid \varphi_{2}(x)=\varphi_{2}(h)=\alpha_{5}\right\}=\{h, j\}=[j]_{\varphi_{2}}} \\
{[i]_{\varphi_{2}}=\left\{x \in \mathcal{O} \mid \varphi_{2}(x)=\varphi_{2}(i)=\alpha_{6}\right\}=\{i\} .}
\end{gathered}
$$

We attain that $\xi_{\varphi_{2}}=\left\{[a]_{\varphi_{2}},[b]_{\varphi_{2}},[e]_{\varphi_{2}},[h]_{\varphi_{2}},[i]_{\varphi_{2}}\right\}$.

$$
[a]_{\varphi_{3}}=\left\{x \in \mathcal{O} \mid \varphi_{3}(x)=\varphi_{3}(a)=\alpha_{2}\right\}=\{a, c\}
$$

$$
\begin{gathered}
=[c]_{\varphi_{3}}, \\
{[b]_{\varphi_{3}}=\left\{x \in \mathcal{O} \mid \varphi_{3}(x)=\varphi_{3}(b)=\alpha_{3}\right\}=\{b, d\}} \\
=[d]_{\varphi_{3}}, \\
{[e]_{\varphi_{3}}=\left\{x \in \mathcal{O} \mid \varphi_{3}(x)=\varphi_{3}(e)=\alpha_{4}\right\}=\{e, g\}} \\
=[g]_{\varphi_{3}}, \\
\quad[f]_{\varphi_{3}}=\left\{x \in \mathcal{O} \mid \varphi_{3}(x)=\varphi_{3}(f)=\alpha_{5}\right\}=\{f\}, \\
{[h]_{\varphi_{3}}=\left\{x \in \mathcal{O} \mid \varphi_{3}(x)=\varphi_{3}(h)=\alpha_{6}\right\}=\{h, i, j\}} \\
=[i]_{\varphi_{3}}=[j]_{\varphi_{3}} .
\end{gathered}
$$

From here, we get that $\xi_{\varphi_{3}}=\left\{[a]_{\varphi_{3}},[b]_{\varphi_{3}},[e]_{\varphi_{3}},[f]_{\varphi_{3}},[h]_{\varphi_{3}}\right\}$. Consequently, a set of partitions of $\mathcal{O}$ is $N_{r}(B)=\left\{\xi_{\varphi_{1}}, \xi_{\varphi_{2}}, \xi_{\varphi_{3}}\right\}$ for $r=1$. Hence, 


$$
\begin{aligned}
& N_{1}(B)^{*} S=\bigcup_{[x]_{\varphi_{i}} \cap S \neq \varnothing}[x]_{\varphi_{i}} \\
& \quad=[b]_{\varphi_{1}} \cup[c]_{\varphi_{1}} \cup[a]_{\varphi_{2}} \cup[b]_{\varphi_{2}} \cup[e]_{\varphi_{2}} \cup[a]_{\varphi_{3}} \cup[b]_{\varphi_{3}} \cup \\
& {[b]_{\varphi_{3}}=\{a, b, c, d, e, f, g\} .}
\end{aligned}
$$

Taking operation tables for $S$ in the tables below:

\begin{tabular}{c|llll}
+ & $b$ & $c$ & $d$ & $e$ \\
\hline$b$ & $c$ & $d$ & $e$ & $f$ \\
$c$ & $d$ & $e$ & $f$ & $g$ \\
$d$ & $e$ & $f$ & $g$ & $a$ \\
$e$ & $f$ & $g$ & $a$ & $b$
\end{tabular}

\begin{tabular}{c|llll}
$\cdot$ & $b$ & $c$ & $d$ & $e$ \\
\hline$b$ & $b$ & $c$ & $d$ & $e$ \\
$c$ & $c$ & $e$ & $g$ & $b$ \\
$d$ & $d$ & $g$ & $c$ & $f$ \\
$e$ & $e$ & $b$ & $f$ & $c$
\end{tabular}

In this case, $(S,+, \cdot)$ is a nearness semiring. Let take $M=\{b, c, d\}$ is subset of $S$.

$$
\begin{aligned}
N_{1}(B)^{*} M= & \bigcup_{[x]_{\varphi_{i}} \cap Q \neq \varnothing}[x]_{\varphi_{i}} \\
& =[b]_{\varphi_{1}} \cup[c]_{\varphi_{1}} \cup[a]_{\varphi_{2}} \cup[b]_{\varphi_{2}} \cup[a]_{\varphi_{3}} \cup[b]_{\varphi_{3}}=
\end{aligned}
$$

$\{\mathrm{a}, b, c, d, e, g\}$.

Since $f \in M S M$ and $f \notin N_{r}(B)^{*} M, M S M \nsubseteq N_{r}(B)^{*} M$. Thus, $M$ is not a bi ideal of nearness semiring $S$.

\section{Theorem 3.4}

Let $S$ be a nearness semiring. If $S$ is commutative and $N_{r}(B)^{*}\left(N_{r}(B)^{*} A\right)=N_{r}(B)^{*} A$, then the product of two quasi ideals of $S$ is a bi ideal of $S$.

Proof. Let $A_{1}$ and $A_{2}$ be quasi ideals of $S$. We show that $\left(A_{1} A_{2}\right) S\left(A_{1} A_{2}\right) \subseteq N_{r}(B)^{*}\left(A_{1} A_{2}\right)$.

We attain that $\left(A_{1} A_{2}\right) S\left(A_{1} A_{2}\right) \subseteq\left(A_{1} A_{2}\right) S\left(S A_{2}\right) \subseteq$ $\left(A_{1} A_{2}\right) S\left(N_{r}(B)^{*} A_{2}\right)$ from Lemma 1. Afterward, $\left(A_{1} A_{2}\right) S\left(N_{r}(B)^{*} A_{2}\right)=A_{1} A_{2} S\left(N_{r}(B)^{*} A_{2}\right) \subseteq$

$\left(N_{r}(B)^{*} A_{1}\right)\left(N_{r}(B)^{*} A_{2}\right) S\left(N_{r}(B)^{*} A_{2}\right) \subseteq$

$\left(N_{r}(B)^{*} A_{1}\right)\left(N_{r}(B)^{*} A_{2}\right)$ by Corollary 1 .

In this case, $\left(N_{r}(B)^{*} A_{1}\right)\left(N_{r}(B)^{*} A_{2}\right) \subseteq N_{r}(B)^{*}\left(A_{1} A_{2}\right)$ by Theorem 2.(ii). Hence, $\left(A_{1} A_{2}\right) S\left(A_{1} A_{2}\right) \subseteq N_{r}(B)^{*}\left(A_{1} A_{2}\right)$ and the product of two quasi ideals of $S$ is a bi ideal of $S$.

\section{Definition 3.10}

Let $S$ be a nearness semiring. $A$ is called nearness $m$-bi ideal of $S$ if $A$ is subsemigroup of $S$ and $A S^{m} A \subseteq N_{r}(B)^{*} A$, where $m$ is pozitive integer and not necessarily 1.

\section{Definition 3.11}

Let $S$ be a nearness semiring. $Q$ is called nearness $(m, n)$-quasi ideal of $S$ if $Q$ is subsemigroup of $S$ and $Q S^{m} \cap S^{n} Q \subseteq N_{r}(B)^{*} Q$, where $m, n$ are pozitive integers.

\section{Theorem 3.5}

Let $S$ be a nearness semiring. Each nearness $(m+1, m+1)$ quasi ideal of $S$ is $m$-bi ideal of $S$.

Proof. Let $S$ be a nearness semiring and $A$ be a $(m+1, m+1)$ quasi ideal of $S$. In this case, it is attainned that $A S^{m} A \subseteq A S^{m} S=$ $A S^{m+1}$ and $A S^{m} A \subseteq S S^{m} A=S^{m+1} A$. Thus, $A S^{m} A \subseteq A S^{m+1} \cap$ $S^{m+1} A$. Since $A$ is a $(m+1, m+1)$-quasi ideal of $S$, we get that $A S^{m} A \subseteq A S^{m+1} \cap S^{m+1} A \subseteq N_{r}(B)^{*} A$. Hence $A S^{m} A \subseteq$ $N_{r}(B)^{*} A$ and $A$ is $m$-bi ideal of $S$.

\section{Conclusion}

As a recent study of nearness semirings, it is defined that the notion of bi ideals in nearness semirings. Afterward, it is explained that some of the concepts and definitions and an example is given with related to the subject. Furthermore, it is given that the definition of nearness $\boldsymbol{m}$-bi ideals and nearness $(\boldsymbol{m}, \boldsymbol{n})$-quasi ideals. And, it is examined that the relationship between them. We believe that these properties will be more useful theoretical development for nearness semiring theory.

\section{References}

[1] Pawlak, Z. (1982). Rough sets, Int. J. Comput. Inform. Sci. 11 (5), 341-356,

[2] Peters, J. F. (2007). Near sets, General theory about nearness of

objects, Appl. Math. Sci. 1 (53-56), 2609-2629.

[3] Peters, J. F. (2007). Near sets, Special theory about nearness of objects, Fund. Inform. 75 (1-4), 407-433.

[4] Peters, J. F. (2013). Near sets: An introduction, Math. Comput. Sci. 7 (1),3-9

[5] İnan, E. and Öztürk, M. A. (2012). Near groups on nearness approximation spaces, Hacet. J. Math. Stat. 41 (4), 545-558.

[6]Öztürk, M. A. (2018)Semiring on weak nearness approximation spaces, Ann. Fuzzy Math. Inform, 15(3), 227241.

[7] Öztürk, M. A. And Temur, İ. (2019). Prime ideals of nearness semirings, Commun. Fac. Sci. Univ. Ank. Ser. Al Math. Stat. 68(2), 1867-1878.

[8] Öztürk, M. A. and Bekmezci, İ.H. (2020). Gamma nearness semirings, Southeast Asian Bull. Math. 44(4), 567-586.

[9] Vandier, H.S.(1934). Note on a simple type of algebra in which cancellation law of addition does not hold, Bull. Am. Math. Soc., 40(12),914-920.

[10] Shabir, A. M and Batod, A. (2004). A note on quasi ideal in semirings, Southeast Asian Bull. Math., 27(5), 923-928.

[11] Good, R.A. and Hughes, D.R.(1952). Associated groups for a semigroups, Bull. Am. Math. Soc., 58 (6), 624-625.

[12] Lajos, S. and Szasz, F.A. (1970) On the bi-ideals in associative ring, Proc. Japan Acad., 46 (6), 505-507.

[13] Tekin, Ö. (2021). Quasi Ideals of Nearness Semirings, Cumhuriyet Sci. J. 42(2), 333-338.

[14] Öztürk, M. A., Jun, Y. B. and İz, A. (2019). Gamma semigroups on weak nearness approximation spaces, J. Int. Math. Virtual Inst. 9 (1), 53-72.

[15] El-Madhoun, N. R. (2007). Quasi ideals and bi-ideals on semigroups and semirings, MSc Thesis, Department of Mathematics, Faculty of Science, The Islamic University of Gaza.

[16] Golan, J. S. (1999). Semirings and Their Applications, Kluwer Academic Publishers. 